\title{
Brain Topography
}

\section{Ipsilateral alteration of resting state activity suggest that cortical dysfunction contributes to the pathogenesis of cluster headache \\ --Manuscript Draft--}

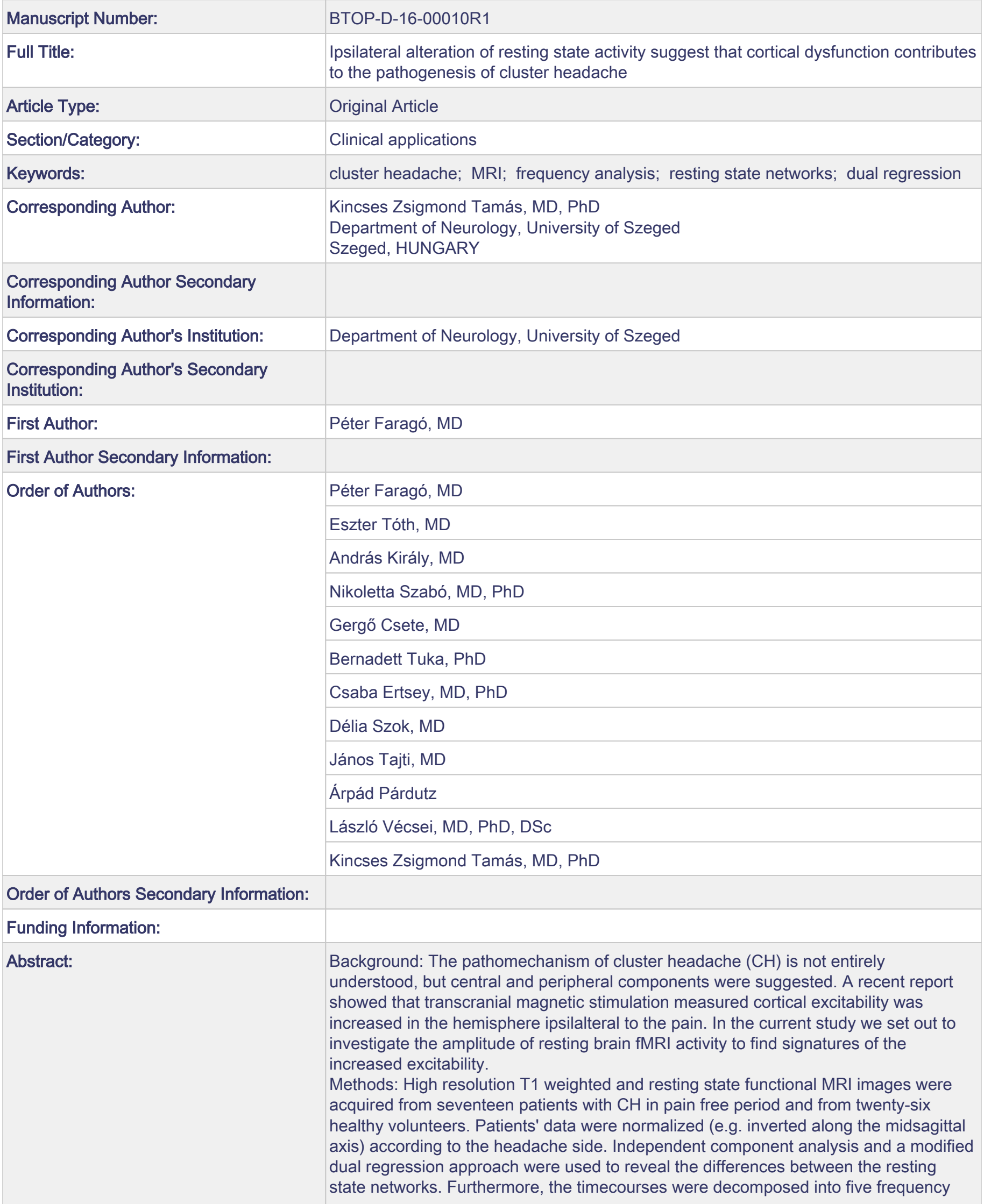


bands by discrete wavelet decomposition and were also re-regressed to the original data to reveal frequency specific resting activity maps.

Results: Two of the identified resting state networks showed alterations in $\mathrm{CH}$. When the data were inverted to have patients' headaches on the left, the ipsilateral attention network showed increased connectivity in $0.08-0.04 \mathrm{~Hz}$ frequency band in the in $\mathrm{CH}$ group. In the same dataset, cerebellar network showed higher functional connectivity in $0.02-0.01 \mathrm{~Hz}$ range in the ipsilateral cerebellum. When the data of patients having headache on the left were inverted to the right, similar increased signal was found in the ipsilateral attention network in $0.08-0.04 \mathrm{~Hz}$ band. The cerebellar network showed increased connectivity in the cerebellum in $0.02-0.01 \mathrm{~Hz}$ band in patients. The Fourier analysis of these area revealed increased power in $\mathrm{CH}$ at all cases.

Conclusions: Our results showed alterations of brain functional networks in $\mathrm{CH}$. The alterations of resting state activity were found in the hemisphere ipsilateral to the pain, signifying the altered cortical processing in the pathomechanism of $\mathrm{CH}$. 


\section{Ipsilateral alteration of resting state activity suggests that cortical dysfunction contributes to the pathogenesis of cluster headache}

Péter Faragó ${ }^{1}$, Nikoletta Szabó ${ }^{1,2}$, Eszter Tóth ${ }^{1}$, Bernadett Tuka ${ }^{1}$, András Király ${ }^{1}$, Gergő Csete ${ }^{1}$, Árpád Párdutz ${ }^{1}$, Délia Szok ${ }^{1}$, János Tajti ${ }^{1}$,Csaba Ertsey ${ }^{3}$, László Vécsei $^{1,4}$, Zsigmond Tamás Kincses ${ }^{1,2}$

${ }^{1}$ Department of Neurology, Faculty of General Medicine, University of Szeged, Szeged, Hungary

${ }^{2}$ International Clinical Research Center, St. Anne's University Hospital Brno, Brno, Czech Republic

${ }^{3}$ Department of Neurology, Semmelweis University, Budapest, Hungary

${ }^{4}$ MTA-SZTE Neuroscience Research Group, Szeged, Hungary

Keywords: cluster headache, MRI, resting state networks, dual regression, frequency analysis

Corresponding author:

Dr. Zsigmond Tamás Kincses

Department of Neurology

Albert Szent-György Clinical Center

University of Szeged

Semmelweis u. 6

6724 - Szeged

Hungary

Email.: kincses.zsigmond.tamas@ med.u-szeged.hu 


\section{Abstract}

Background: The pathomechanism of cluster headache $(\mathrm{CH})$ is not entirely understood, but central and peripheral components were suggested. A recent report showed that transcranial magnetic stimulation measured cortical excitability was increased in the hemisphere ipsilalteral to the pain. In the current study we set out to investigate the amplitude of resting brain fMRI activity to find signatures of the increased excitability. Methods: High resolution T1 weighted and resting state functional MRI images were acquired from seventeen patients with $\mathrm{CH}$ in pain free period and from twenty-six healthy volunteers. Patients' data were normalized (e.g. inverted along the midsagittal axis) according to the headache side. Independent component analysis and a modified dual regression approach were used to reveal the differences between the resting state networks. Furthermore, the timecourses were decomposed into five frequency bands by discrete wavelet decomposition and were also re-regressed to the original data to reveal frequency specific resting activity maps.

Results: Two of the identified resting state networks showed alterations in $\mathrm{CH}$. When the data were inverted to have patients' headaches on the left, the ipsilateral attention network showed increased connectivity in $0.08-0.04 \mathrm{~Hz}$ frequency band in the in $\mathrm{CH}$ group. In the same dataset, cerebellar network showed higher functional connectivity in $0.02-0.01 \mathrm{~Hz}$ range in the ipsilateral cerebellum. When the data of patients having headache on the left were inverted to the right, similar increased signal was found in the ipsilateral attention network in $0.08-0.04 \mathrm{~Hz}$ band. The cerebellar network showed increased connectivity in the cerebellum in $0.02-0.01 \mathrm{~Hz}$ band in patients. The Fourier analysis of these area revealed increased power in $\mathrm{CH}$ at all cases. 
Conclusions: Our results showed alterations of brain functional networks in $\mathrm{CH}$. The alterations of resting state activity were found in the hemisphere ipsilateral to the pain, signifying the altered cortical processing in the pathomechanism of $\mathrm{CH}$. 


\section{Introduction}

Cluster headache $(\mathrm{CH})$ is one of the most painful primary headache disorders. The disease is characterized by severe, strictly unilateral, retro-orbital pain with ipsilateral autonomic symptoms, such as lacrimation, rhinorrhea, conjunctival injection (Headache Classification Committee of the International Headache 2013). These attacks usually appear on a daily basis for a period lasting several weeks or months (the cluster period), followed by lengthy headache-free periods. The cluster periods notably follow a circadian pattern. While there are some effective possibilities for acute treatment of attacks, a highly effective preventative measure is missing.

Imaging studies provided several clues about the disease. Structural imaging studies revealed, the total gray matter volume was decreased in $\mathrm{CH}$ patients compared to healthy controls in the frontal lobe in interictal period (Yang et al. 2013). We and others showed that there is an extensive white matter microstructural alteration in the pain free period in CH (Szabo et al. 2013; Teepker et al. 2012).

Functional imaging studies showed higher activation in the ipsilateral hypothalamus during attacks with positron emission tomography (May et al. 1998) and functional MRI (May et al. 2000; Morelli et al. 2009; Sprenger et al. 2004). Furthermore, altered functional connectivity of the hypothalamus and anterior thalamus were also described (Qiu et al. 2015; Qiu et al. 2013; Rocca et al. 2010; Yang et al. 2014). Moreover, the activity of the pain matrix (e.g. according to Tracey: „large distributed brain network [activating] during nociceptive processing" (Tracey 2008)) and the connectivity to some of its structures were found to be altered too (Sprenger et al. 2007; Yang et al. 2014).

The alteration of the functional connectivity between certain nodes or the expression of spatially distributed functional networks can be deciphered from the resting state 
functional MRI (Beckmann et al. 2005b; Fox and Raichle 2007). The alterations of these functional networks were shown in several diseases such as multiple sclerosis (Roosendaal et al. 2010), migraine (Xue et al. 2012) or Alzheimer's disease (Greicius et al. 2004). Most of the approaches analyzing the resting state BOLD fluctuations focus on the correlated activity in various brain regions or search for spatially independent common temporal characteristics in the fMRI data. The information in the amplitude and the frequency of these resting activity fluctuations only recently came into the focus of attention (Kublbock et al. 2014; Zou et al. 2008). However, none of the previous studies examined alterations between the resting state networks' power and their relation to the pain matrix in cluster headache. The in depth analysis of such information of the resting state fluctuation is especially important in the view of recent researches showing an increased cortical excitability in $\mathrm{CH}$ (Cosentino et al. 2015) similar to that in migraine (Chadaide et al. 2007). While there is no direct evidence that various parameters of TMS measured cortical excitability are represented as a variation in the amplitude or the frequency of the resting BOLD fluctuations, one might speculate that they are strongly related and both depict important features of cortical function.

In this study we set out to investigate resting state fMRI networks in cluster headache patients in the pain free period with a special attention to the amplitude of the resting activity in various frequency bands. According to our hypothesis, an altered resting activity might be responsible for the development of pain, or alternatively repeated pain bouts may cause maladaptive plastic changes in the brain that can be detected in the resting functional connectivity and also in the local brain activity. Furthermore, these changes can potentially be linked to clinical data, such as disease duration or cumulative headache days. 


\section{Methods}

\section{Participants}

Seventeen patients with episodic $\mathrm{CH}$ were recruited to this study from the Headache Outpatients Clinics of the University of Szeged and Semmelweis University. The diagnosis of the $\mathrm{CH}$ was made according to the International Classification of Headache Disorders. The participants had no history of any other neurological or psychiatric disorders. None of the participants used prophylactic interval therapy. The MRI scans were acquired at least 1 month after the last headache episode. Clinical variables, such as disease duration, time between bouts and average length of bouts was recorded for all patients. Furthermore, cumulative headache days was estimated for all patients that is the total number of days the patient had experienced headache over his/her entire life. Twenty-six age and sex matched healthy individuals were recruited as a control group. All participants were right handed. The demographic data is depicted in Table 1. The study was approved by the ethics committee of the University of Szeged and all study participants gave written informed consent in accordance with the Declaration of Helsinki (authority number: 56/2011).

\section{Image acquisition}

The MR imaging was performed on a 1.5T GE Signa Excite HDxt MRI Scanner (Milwaukee, WI, USA). The head motion was restricted with foam padding around the head and the noise of the scanner was attenuated with earplugs. For every participant high resolution T1 weighted images (3D IR-FSPGR: TR/TE/TI: 10.3/4.2/450ms, flip angle: $15^{\circ}$, ASSET: $2, \mathrm{FOV}: 25 * 25 \mathrm{~cm}$, matrix: $256 * 256$, slice thickness: $1 \mathrm{~mm}$ ) and a resting state fMRI protocol with echo-planar imaging technique (TE: $40 \mathrm{~ms}$, TR: 300 
ms, matrix: $64 * 64 \mathrm{~cm}, \mathrm{FOV}: 30 * 30 \mathrm{~cm}$, slice thickness: $6 \mathrm{~mm}$, flip angle: $90^{\circ}$, NEX: 1, ASSET: 2,0 Ph, Phases per Loc: 128, volumes: 200) were acquired. Subjects were asked to stay awake during the acquisition and lay in the scanner with closed eyes.

\section{Data preprocessing}

All image processing was carried out with the algorithms in FMRIB's Software Library (http://www.fmrib.ox.uk/fsl, Oxford, UK). Since in $\mathrm{CH}$ the headache is strictly unilateral, according to the literature (Absinta et al. 2012; Szabo et al. 2013; Yang et al. 2014), we normalized the data according to the headache side: the images of patients having headache on the left side were inverted along the midsagittal axis by the fslswapdim command. To acknowledge that there are non-pain related differences between the left and right hemispheres we repeated the analysis by inverting the images of the patients who had headache on the right side. (The analysis of the noninverted data is presented in the Supplementary material.) The pre-processing was carried out with FEAT (FMRI Expert Analysis Tool). The first two non steady-state volumes from the 200 resting-state images were wiped out. Brain extraction (BET) was applied in all structural and functional images to remove all non-brain tissues (Smith 2002). The images were motion corrected by MCFLIRT and spatially smoothed with Gaussian kernel of 6 mm FWHM (Jenkinson et al. 2002). A high-pass filter with cutoff sigma of $100 \mathrm{~s}$ was applied to all functional images.

Furthermore, all participants' functional images were registered to their own T1 images with boundary-based registration and to a standard brain (MNI152T1) with a linear registration with 12 degree of freedom using FLIRT and then a non-linear registration via FSL's FNIRT (Jenkinson et al. 2002). The functional data were transformed to a standard space with $4 \mathrm{~mm}$ resampling resolution. 


\section{Task-free network identification}

Independent component analysis was used to identify the large-scale neuronal networks (Beckmann et al. 2005b). The investigation was carried out with Multivariate Exploratory Linear Optimized Decomposition into Independent Components (MELODIC) command using multi-session temporal concatenation.

The preprocessed data variance was normalized and demeaned, and then a voxel wise concatenation was applied. The resulted dataset was decomposed into a set of independent components that characterize the underlying processes in the spatial and temporal domains in such a way that spatial matrices are maximally non-Gaussian. The spatial maps of the resting state networks were thresholded to $\mathrm{p}<0.5$ by using Gaussian/Gamma mixture model fitting as an alternative hypothesis.

Spatio-temporal components representing artifacts, outliers and non-pain related networks were excluded from further analysis (Beckmann et al. 2005b; Qiu et al. 2013; Tian et al. 2013; Yang et al. 2014). Eight networks were subjected to further analysis: medial visual network, lateral visual network, auditory network, sensorimotor network, default mode network, salience network, ipsilateral and contralateral attention network, cerebellar network (Beckmann et al. 2005a).

\section{Between group differences of the expression of the resting state networks}

The analysis of between group differences were carried out by a modified dual regression approach (Beckmann C. F. 2009; Filippini et al. 2009). The selected networks' spatial map was thresholded at a probability greater than 0.5 and binarised. The maps were than transformed into the individuals' resting fMRI space to reveal the subject specific temporal dynamics of the networks. 
These time courses were decomposed by discrete wavelet decomposition (see below) into five frequency bands. Then the band-pass filtered timecourses were used in a linear model fit (temporal regression) against the associated fMRI data to estimate subjectand frequency-specific spatial maps. Finally, these spatial maps were compared across groups. Modelling was accomplished using standard general linear model (GLM) setup, explanatory variable coded for group membership. To examine the correlation between the clinical variables and the frequency specific expression of resting state networks cumulative headache days were used as a regressor in the GLM design. Statistical inference was carried out by using nonparametric permutation test (5000 permutations) (Nichols and Holmes 2002). Thresholding was carried out with threshold-free cluster enhancement (TFCE) (Smith and Nichols 2009); the statistical results were corrected for multiple comparisons. (The scheme of the modified dual regression analysis is depicted in the Supplementary material.)

In order to restrict the dual regression analysis to specific frequency bands discrete wavelet decomposition of the time courses was used to bandpass filter the data before the second regression step. (Wavelet Toolbox of the Matlab software package; MathWorks Inc). The discrete wavelet decomposition is an implementation of the wavelet transform using a set of predefined wavelet scales and translations and decomposes the signal into mutually orthogonal set of wavelets. Wavelets are brief waves, they are finitely extended and their oscillations decay to zero rapidly, satisfying the admissibility condition:

$\int \Psi(t) d t=0$.

By dilating and translating a "mother" wavelet $(\Psi)$ and a "father" wavelet $(\Phi)$

$\int \Phi(t) d t=1$

a wavelet family can be obtained: 


$$
\Psi_{j k}(t)=\frac{1}{\sqrt{2^{j}}} \Psi\left(\frac{t-2^{j} k}{2^{j}}\right)
$$$$
\Phi_{j k}(t)=\frac{1}{\sqrt{2^{j}}} \Phi\left(\frac{t-2^{j} k}{2^{j}}\right)
$$

where $\mathrm{j}$ is index of the scale $S_{j}=2^{j}$ and k indexes the $K=n / 2^{j}$ location in time. The Daubechies wavelet was used as mother wavelet. The analysis by using a halfband filtering, decomposes the data over a hierarchy of scales $\left(S_{j}\right)$. At each scale the data is split into two orthogonal components: details $\left(d_{j k}\right)$ containing the high frequency information and approximations $\left(a_{j k}\right)$ containing the low frequency information. By five levels of decomposition the following frequency bands were derived: 0-0.16 Hz: 0.16$0.08 \mathrm{~Hz}, 0.08-0.04 \mathrm{~Hz}, 0.04-0.02 \mathrm{~Hz}, 0.02-0.01 \mathrm{~Hz}$ and $0.01-0 \mathrm{~Hz}$. These decomposed, band-pass filtered timecourses were used in the modified dual regression approach (see above).

\section{Amplitude of the resting state activity}

For each subject the mean time course of every resting state network where group differences were detected with the dual regression analysis was extracted and subjected to further analysis. To investigate the amplitude of the resting activity in the different frequency bands the individual time courses of each of the subjects for the components of interest were subjected to Fourier Transformation. The power spectrums were compared between groups by two-sample t-tests and the results were corrected for multiple comparisons by Bonferoni correction. 


\section{Results}

\section{Network identification}

The ICA analysis identified 30 different spatio-temporal components in the right inverted dataset and 29 in the left inverted dataset. Resting state networks revealed by previous articles showed similar distribution (Beckmann et al. 2005b; Mantini et al. 2007). Based on these articles, we selected six of these networks for further analysis: default mode network, contralateral and ipsilateral attention network, medial visual network, lateral visual network, cerebellar network.

\section{Frequency specific expression of resting state networks}

\section{Left sided headache dataset}

The dual regression analysis revealed alterations of the ipsilateral attention network in $\mathrm{CH}$ patients in two frequency bands: $0.08-0.04 \mathrm{~Hz}$ and $0.04-0.02 \mathrm{~Hz}$. A significantly increased degree of coactivation within the ipsilateral attention network was found in the superior frontal gyrus and the medial frontal cortex $(p<0.03$; Figure 1/a and Table 2).

A significantly increased degree of coactivation within the cerebellar network was found in $\mathrm{CH}$ patients in the $0.02-0.01 \mathrm{~Hz}$ frequency range in the ipsilateral cerebellar hemisphere ( $\mathrm{p}<0.03$; Figure $1 / \mathrm{b}$ and Table 2$)$.

The Fourier Transformation revealed increased power activity in patients of the ipsilateral attention network in the $0.04-0.07 \mathrm{~Hz}$ frequency band. Similarly, in the cerebellar network between $0-0.02 \mathrm{~Hz}$ patients showed a higher activity (Figure 1/c). 
There were no other significant differences between the groups in other resting state networks in any other frequency bands.

\section{Right sided headache data set}

A significantly increased degree of coactivation within the ipsilateral attention network was found in patients in the $0.04-0.08 \mathrm{~Hz}$ frequency band in the right superior frontal gyrus $(p<0.003)$ and in the right medial frontal gyrus $(p<0.05$; Figure $2 / a$ and Table 2$)$. In the cerebellar network, in the frequency range of $0.01-0.02 \mathrm{~Hz}$, a significantly increased degree of coactivation was found in the contralateral cerebellum in the $\mathrm{CH}$ group compared to controls ( $\mathrm{p}<0.05$; Figure $2 / \mathrm{b}$ and Table 2).

The power spectrum analysis showed higher activity at the ipsilateral attention network between $0.04-0.06 \mathrm{~Hz}$ and at the cerebellar network around $0.02 \mathrm{~Hz}$ (Figure 2/c).

There were no differences between the control and patient group in any other networks in any of the frequency bands.

\section{Connection between resting state activity and clinical data}

The correlation between the cumulative headache days and degree of coactivation in the resting state networks revealed significant results in one network in each dataset (right and left sided headache dataset).

The analysis of the left sided headache dataset revealed negative correlation between the cumulative headache days and the degree of coactivation in the contralateral attention network in the $0.04-0.08 \mathrm{~Hz}$ band in the contralateral frontal pole $(\mathrm{R}=-0.83$, $\mathrm{p}<0.001$; Figure 3/a).

The analysis of the right sided headache dataset revealed negative correlation between the degree of coactivation within the contralateral attention network and the cumulative 
number of the headache days in the $0.16-0.08 \mathrm{~Hz}$ frequency band in the contralateral frontal pole $(\mathrm{R}=-0.81 ; \mathrm{p}<0.05$; Figure $3 / \mathrm{b})$.

There was no other correlation between the cumulative headache days and degree of coactivation in any other resting state network. No other clinical variables showed significant correlation with the measured MRI parameters.

\section{Discussion}

Our study revealed increased frequency specific activity in $\mathrm{CH}$ patients in the attention network ipsilateral to the headache side and in the contralateral cerebellar network. This increased activity must be understood as increased frequency specific coherent activity within networks and also increased amplitude of the BOLD fluctuation.

Former studies agreed on the altered functional connectivity of the hypothalamus in $\mathrm{CH}$ (Qiu et al. 2015; Qiu et al. 2013; Yang et al. 2014), and also on the alteration of the connectivity of other regions such as the medial frontal cortex and the cerebellum. Similarly, Rocca and co-workers found decreased resting BOLD fluctuation in the sensorimotor network in $\mathrm{CH}$ with some of which regions are also in the medial frontal cortex (Rocca et al. 2010). Our results also pointed out the altered connectivity of the medial prefrontal region. The altered resting state activity of the medial frontal cortex, which is part of various resting state networks points to the key role of the medial frontal cortex in the pain perception or in the pathogenesis of $\mathrm{CH}$.

Our results also fit well into our current knowledge about $\mathrm{CH}$, hypothalamus being a key component of the pathomechanism. The anteroventral compartment of the hypothalamus was shown to be connected predominantly with the prefrontal cortex and the dorsal supplementary motor area in a diffusion tractography study (Lemaire et al. 2011). Similar hypothalamo-cortical connections were found in rodents (Risold et al. 
1997). On the other hand cerebellar connection of the hypothalamus was also revealed in humans (Lemaire et al. 2011) and in animal studies (Dietrichs and Haines 2002). Moreover the effective site of the deep brain stimulation for $\mathrm{CH}$ in the ipsilateral hypothalamus was shown to have high probability connectivity to the medial lemniscus, frontal cortex and to the cerebellum (Owen et al. 2007).

Furthermore, the frontal regions that showed altered resting BOLD fluctuations are very much alike to some of the regions showing atrophy in $\mathrm{CH}$ (Absinta et al. 2012; Naegel et al. 2014). Furthermore, the disorganized connectivity could be a result of white matter microstructural alteration that we have described in $\mathrm{CH}$ earlier (Szabo et al. 2013).

We also found a higher amplitude activity in patients in areas where we found higher connectivity. Former studies investigated the amplitude of the resting state fluctuation in diseases with chronic pain (Baliki et al. 2014; Malinen et al. 2010b). Baliki and colleagues found increased amplitude of activity in the default mode network in patients with chronic pain. The alterations were found predominantly in the higher frequencies. This article also describes increased amplitude of BOLD activity in a pain related network and in the cerebellum.

One important aspect of our results is that the medial frontal activity differences were in the attention network ipsilateral to the headache side, a result coincide with former transcranial magnetic stimulation study showing increased cortical excitability ipsilateral to the pain in $\mathrm{CH}$ (Cosentino et al. 2015). While several studies indicated the important role of the hypothalamus in CH (May et al. 1998; May et al. 2000; Morelli et al. 2009; Qiu et al. 2015; Qiu et al. 2013; Rocca et al. 2010; Sprenger et al. 2004; Yang et al. 2014), it is still debated if it actively contributes to the attacks or the altered hypothalamic activation is secondary to the cortical and subcortical malfunction of the 
pain matrix. The primary role of the cortical dysfunction was strengthened by former electrophysiological studies (Casale et al. 2008; van Vliet et al. 2003) and the existence of aura symptoms before the pain also point to this direction. The increased cortical excitability (Cosentino et al. 2015) might present in an increased amplitude of BOLD fluctuation-and might lead to the changes of various transmitter levels that activates the trigeminal endings such as in migraine (Tajti et al. 2015; Tuka et al. 2013).

Incontrast to the ipsilateral functional alterations found in the current investigation, microstructural alterations in our previous DTI study were somewhat lateralized to the hemisphere contralateral to the headache (Szabo et al. 2013) suggesting chronic microstructural alterations, which we believe is a kind of a disintegration of the white matter due to the recurring painful attacks.

Recently, the focus of attention has shifted to investigate the activity of resting state fluctuations in various frequency bands (Gao et al. 2015), especially in pain conditions (Kim et al. 2013; Otti et al. 2013). The importance of the various frequencies of BOLD fluctuations is not yet known. It was proposed that functional connectivity of various brain regions are represented in different dominant frequency bands (Salvador et al. 2008). This could be responsible for the alterations found in different frequency bands in the attention network and the cerebellar network. Another option might be that $\mathrm{CH}$ was proposed to be a neurovascular disease, and the altered neurovascular coupling may affect the frequency of the resting BOLD fluctuations (Malinen et al. 2010a; Malinen et al. 2010b) by acting as a filter. Furthermore, the group difference in the resting state network activity might well be the result of improved signal to noise ratio by filtering out the low and high frequency artifact. Since most of the slow frequency fluctuation in our analysis with a relatively long TR were shown to be neural origin (Boyacioglu et al. 2013), this hypothesis seems rather unlikely. 


\section{Conclusion}

We propose that the altered resting state activity on the side of headache that might be a signature of increased cortical excitability may play an important role in the pathogenesis of $\mathrm{CH}$.

\section{Conflict of interest}

The authors declare that there are no conflicts of interest.

\section{Acknowledgement}

The study was supported by the „Neuroscience Research Group of the Hungarian Academy of Sciences and University of Szeged", project FNUSA-ICRC (no. CZ.1.05/1.1.00/02.0123) from the European Regional Development Fund, the National Brain Research Program (Grant No. KTIA_13_NAP-A-II/20.) and an OTKA [PD 104715] grant. Dr. Szabó and Dr. Kincses were supported by European Regional Development Fund - Project FNUSA-ICRC (No. CZ.1.05/1.1.00/02.0123) and by European Union - project ICRC-ERA-HumanBridge (No. 316345). Dr. Párdutz was supported by the Bolyai Scholarship Program of the Hungarian Academy of Sciences. 


\section{References}

Absinta M, Rocca MA, Colombo B, Falini A, Comi G, Filippi M (2012) Selective decreased grey matter volume of the pain-matrix network in cluster headache Cephalalgia : an international journal of headache 32:109-115 doi:10.1177/0333102411431334

Baliki MN, Mansour AR, Baria AT, Apkarian AV (2014) Functional Reorganization of the Default Mode Network across Chronic Pain Conditions PloS one 9 doi:ARTN e106133

10.1371/journal.pone.0106133

Beckmann C. F. FN, Smith S.M. (2009) Group comparison of resting-state FMRI data using multi-subject ICA and dual regression. NeuroImage 47:S39-S41

Beckmann CF, DeLuca M, Devlin JT, Smith SM (2005a) Investigations into restingstate connectivity using independent component analysis Philos Trans R Soc Lond B Biol Sci 360:1001-1013 doi:10.1098/rstb.2005.1634

Beckmann CF, DeLuca M, Devlin JT, Smith SM (2005b) Investigations into restingstate connectivity using independent component analysis Philos $\mathrm{T}$ Roy Soc B 360:1001-1013 doi:Doi 10.1098/Rstb.2005.1634

Boyacioglu R, Beckmann CF, Barth M (2013) An Investigation of RSN Frequency Spectra Using Ultra-Fast Generalized Inverse Imaging Frontiers in human neuroscience 7:156 doi:10.3389/fnhum.2013.00156

Casale MS, Baratto M, Cervera C, Gallamini M, Lynch G, Gjini K, Boutros NN (2008) Auditory evoked potential abnormalities in cluster headache Neuroreport 19:16331636 doi:10.1097/WNR.0b013e328314e0dd 
Chadaide Z, Arlt S, Antal A, Nitsche MA, Lang N, Paulus W (2007) Transcranial direct current stimulation reveals inhibitory deficiency in migraine Cephalalgia 27:833-839 doi:10.1111/j.1468-2982.2007.01337.x

Cosentino G, Brighina F, Brancato S, Valentino F, Indovino S, Fierro B (2015) Transcranial magnetic stimulation reveals cortical hyperexcitability in episodic cluster headache J Pain 16:53-59 doi:10.1016/j.jpain.2014.10.006

Dietrichs E, Haines DE (2002) Possible pathways for cerebellar modulation of autonomic responses: micturition Scandinavian journal of urology and nephrology Supplementum:16-20

Filippini $\mathrm{N}$ et al. (2009) Distinct patterns of brain activity in young carriers of the APOE-epsilon4 allele Proc Natl Acad Sci U S A 106:7209-7214 doi:10.1073/pnas.0811879106

Fox MD, Raichle ME (2007) Spontaneous fluctuations in brain activity observed with functional magnetic resonance imaging Nature reviews Neuroscience 8:700-711 doi:10.1038/nrn2201

Gao L et al. (2015) Frequency-dependent changes of local resting oscillations in sleepdeprived brain PloS one 10:e0120323 doi:10.1371/journal.pone.0120323

Greicius MD, Srivastava G, Reiss AL, Menon V (2004) Default-mode network activity distinguishes Alzheimer's disease from healthy aging: evidence from functional MRI Proceedings of the National Academy of Sciences of the United States of America 101:4637-4642 doi:10.1073/pnas.0308627101

Headache Classification Committee of the International Headache S (2013) The International Classification of Headache Disorders, 3rd edition (beta version) Cephalalgia : an international journal of headache 33:629-808 doi:10.1177/0333102413485658 
Jenkinson M, Bannister P, Brady M, Smith S (2002) Improved optimization for the robust and accurate linear registration and motion correction of brain images NeuroImage 17:825-841

Kim JY et al. (2013) Increased power spectral density in resting-state pain-related brain networks in fibromyalgia Pain 154:1792-1797 doi:10.1016/j.pain.2013.05.040

Kublbock M et al. (2014) Stability of low-frequency fluctuation amplitudes in prolonged resting-state fMRI NeuroImage 103C:249-257 doi:10.1016/j.neuroimage.2014.09.038

Lemaire JJ et al. (2011) White matter connectivity of human hypothalamus Brain research 1371:43-64 doi:10.1016/j.brainres.2010.11.072

Malinen S et al. (2010a) Aberrant temporal and spatial brain activity during rest in patients with chronic pain Proceedings of the National Academy of Sciences of the United States of America 107:6493-6497 doi:10.1073/pnas.1001504107

Malinen S et al. (2010b) Aberrant temporal and spatial brain activity during rest in patients with chronic pain Proceedings of the National Academy of Sciences of the United States of America 107:6493-6497 doi:10.1073/pnas.1001504107

Mantini D, Perrucci MG, Del Gratta C, Romani GL, Corbetta M (2007) Electrophysiological signatures of resting state networks in the human brain Proceedings of the National Academy of Sciences of the United States of America 104:13170-13175 doi:10.1073/pnas.0700668104

May A, Bahra A, Buchel C, Frackowiak RS, Goadsby PJ (1998) Hypothalamic activation in cluster headache attacks Lancet 352:275-278 doi:10.1016/S01406736(98)02470-2

May A, Bahra A, Buchel C, Frackowiak RS, Goadsby PJ (2000) PET and MRA findings in cluster headache and MRA in experimental pain Neurology 55:1328-1335 
Morelli N, Pesaresi I, Cafforio G, Maluccio MR, Gori S, Di Salle F, Murri L (2009) Functional magnetic resonance imaging in episodic cluster headache The journal of headache and pain 10:11-14 doi:10.1007/s10194-008-0085-z

Naegel S, Holle D, Desmarattes N, Theysohn N, Diener HC, Katsarava Z, Obermann M (2014) Cortical plasticity in episodic and chronic cluster headache NeuroImage Clinical 6:415-423 doi:10.1016/j.nicl.2014.10.003

Nichols TE, Holmes AP (2002) Nonparametric permutation tests for functional neuroimaging: A primer with examples Hum Brain Mapp 15:1-25 doi:Doi 10.1002/Hbm.1058

Otti A, Guendel H, Wohlschlager A, Zimmer C, Noll-Hussong M (2013) Frequency shifts in the anterior default mode network and the salience network in chronic pain disorder BMC psychiatry 13:84 doi:10.1186/1471-244X-13-84

Owen SL et al. (2007) Connectivity of an effective hypothalamic surgical target for cluster headache Journal of clinical neuroscience : official journal of the Neurosurgical Society of Australasia 14:955-960 doi:10.1016/j.jocn.2006.07.012

Qiu E, Tian L, Wang Y, Ma L, Yu S (2015) Abnormal coactivation of the hypothalamus and salience network in patients with cluster headache Neurology doi:10.1212/WNL.0000000000001442

Qiu E et al. (2013) Abnormal brain functional connectivity of the hypothalamus in cluster headaches PloS one 8:e57896 doi:10.1371/journal.pone.0057896

Risold PY, Thompson RH, Swanson LW (1997) The structural organization of connections between hypothalamus and cerebral cortex Brain research Brain research reviews 24:197-254 
Rocca MA et al. (2010) Central nervous system dysregulation extends beyond the painmatrix network in cluster headache Cephalalgia : an international journal of headache 30:1383-1391 doi:10.1177/0333102410365164

Roosendaal SD, Schoonheim MM, Hulst HE, Sanz-Arigita EJ, Smith SM, Geurts JJG, Barkhof F (2010) Resting state networks change in clinically isolated syndrome Brain : a journal of neurology 133:1612-1621 doi:Doi 10.1093/Brain/Awq058

Salvador R et al. (2008) A simple view of the brain through a frequency-specific $\begin{array}{llll}\text { functional } & \text { connectivity } & \text { 39:279-289 }\end{array}$ doi:10.1016/j.neuroimage.2007.08.018

Smith SM (2002) Fast robust automated brain extraction Human brain mapping 17:143155 doi:10.1002/hbm.10062

Smith SM, Nichols TE (2009) Threshold-free cluster enhancement: addressing problems of smoothing, threshold dependence and localisation in cluster inference NeuroImage 44:83-98 doi:10.1016/j.neuroimage.2008.03.061

Sprenger T, Boecker H, Tolle TR, Bussone G, May A, Leone M (2004) Specific hypothalamic activation during a spontaneous cluster headache attack Neurology $62: 516-517$

Sprenger T et al. (2007) Altered metabolism in frontal brain circuits in cluster headache Cephalalgia : an international journal of headache 27:1033-1042 doi:10.1111/j.14682982.2007.01386.x

Szabo N, Kincses ZT, Pardutz A, Toth E, Szok D, Csete G, Vecsei L (2013) White matter disintegration in cluster headache The journal of headache and pain 14:64 doi:10.1186/1129-2377-14-64

Tajti J, Szok D, Majláth Z, Tuka B, Csáti A, Vécsei L (2015) Migraine and neuropeptides Neuropeptides 52:19-30 doi:10.1016/j.npep.2015.03.006 
Teepker M et al. (2012) Diffusion tensor imaging in episodic cluster headache Headache 52:274-282 doi:10.1111/j.1526-4610.2011.02000.x

Tian L, Kong Y, Ren J, Varoquaux G, Zang Y, Smith SM (2013) Spatial vs. Temporal Features in ICA of Resting-State fMRI - A Quantitative and Qualitative Investigation in the Context of Response Inhibition PloS one 8:e66572 doi:10.1371/journal.pone.0066572

Tracey I (2008) Imaging pain Br J Anaesth 101:32-39 doi:10.1093/bja/aen102

Tuka B et al. (2013) Alterations in PACAP-38-like immunoreactivity in the plasma during ictal and interictal periods of migraine patients Cephalalgia 33:1085-1095 doi:10.1177/0333102413483931

van Vliet JA, Vein A, Le Cessie S, Ferrari MD, van Dijk JG, Group DRR (2003) Impairment of trigeminal sensory pathways in cluster headache Cephalalgia 23:414419

Xue $\mathrm{T}$ et al. (2012) Intrinsic brain network abnormalities in migraines without aura revealed in resting-state fMRI PloS one 7:e52927 doi:10.1371/journal.pone.0052927 Yang FC et al. (2013) Altered gray matter volume in the frontal pain modulation network in patients with cluster headache Pain 154:801-807 doi:10.1016/j.pain.2013.02.005

Yang FC et al. (2014) Altered hypothalamic functional connectivity in cluster headache: a longitudinal resting-state functional MRI study Journal of neurology, neurosurgery, and psychiatry doi:10.1136/jnnp-2014-308122

Zou QH et al. (2008) An improved approach to detection of amplitude of low-frequency fluctuation (ALFF) for resting-state fMRI: fractional ALFF Journal of neuroscience methods 172:137-141 doi:10.1016/j.jneumeth.2008.04.012 


\section{Tables}

Table 1. Demographic data of the cluster headache patients

Table 2. MNI coordinates $(\mathrm{mm})$ of the increased degree of coactivation in cluster headache patients. 


\section{Figure legends}

Figure 1. Altered activity of the left mirrored dataset. The ipsilateral attention network showed increased activity in the ipsilateral superior frontal gyrus and in the ipsilateral medio-frontal cortex (a). The increased activity was measured at the frequency band $0.08-0.04 \mathrm{~Hz}(\mathrm{p}<0.05)$ as shown on the power spectrum below. In the cerebellar network increased activity was found in the cerebellar tonsils at the frequency band $0.02-0.01 \mathrm{~Hz}(\mathrm{p}<0.05)$ as depicted on the power spectrum below. The colorbars above the images represent the p-values. On the power spectrum figures the black curve represents to the $\mathrm{CH}$ group mean amplitude, and red curve stands for the healthy group. The green columns displayed the significant differences between the two groups corrected for multiple comparisons.

Figure 2. Altered activity of the right mirrored dataset. The ipsilateral attention network showed increased activity in the $0.08-0.04 \mathrm{~Hz}$ frequency band $(\mathrm{p}<0.01)$ in the superior and medial frontal cortex. The analysis revealed decreased activity in the cerebellar network in the cerebellar tonsils (b) at the frequency band $0.02-0.01 \mathrm{~Hz}$ $(\mathrm{p}<0.05)$. The colorbars represent $\mathrm{p}$-values. The reduced activity is depicted to blue to light blue. The power spectrum of the resting state network's activity is presented below the statistical images. Black curve stands for the $\mathrm{CH}$ group mean amplitude, and red curve for the healthy group. The green columns indicate the significant differences between the two groups corrected for multiple comparisons.

Figure 3. Correlation was found between cumulative headache days and expression of the resting state activity fluctuations. In the left mirrored dataset, the 
contralateral attention network showed correlation with the cumulative headache days in the contralateral frontal pole $(\mathrm{p}<0.05)$. The right mirrored dataset showed correlation in the contralateral attention network (b) near the ipsilateral frontal pole $(\mathrm{p}<0.05)$. The colorbar represents p-values. 

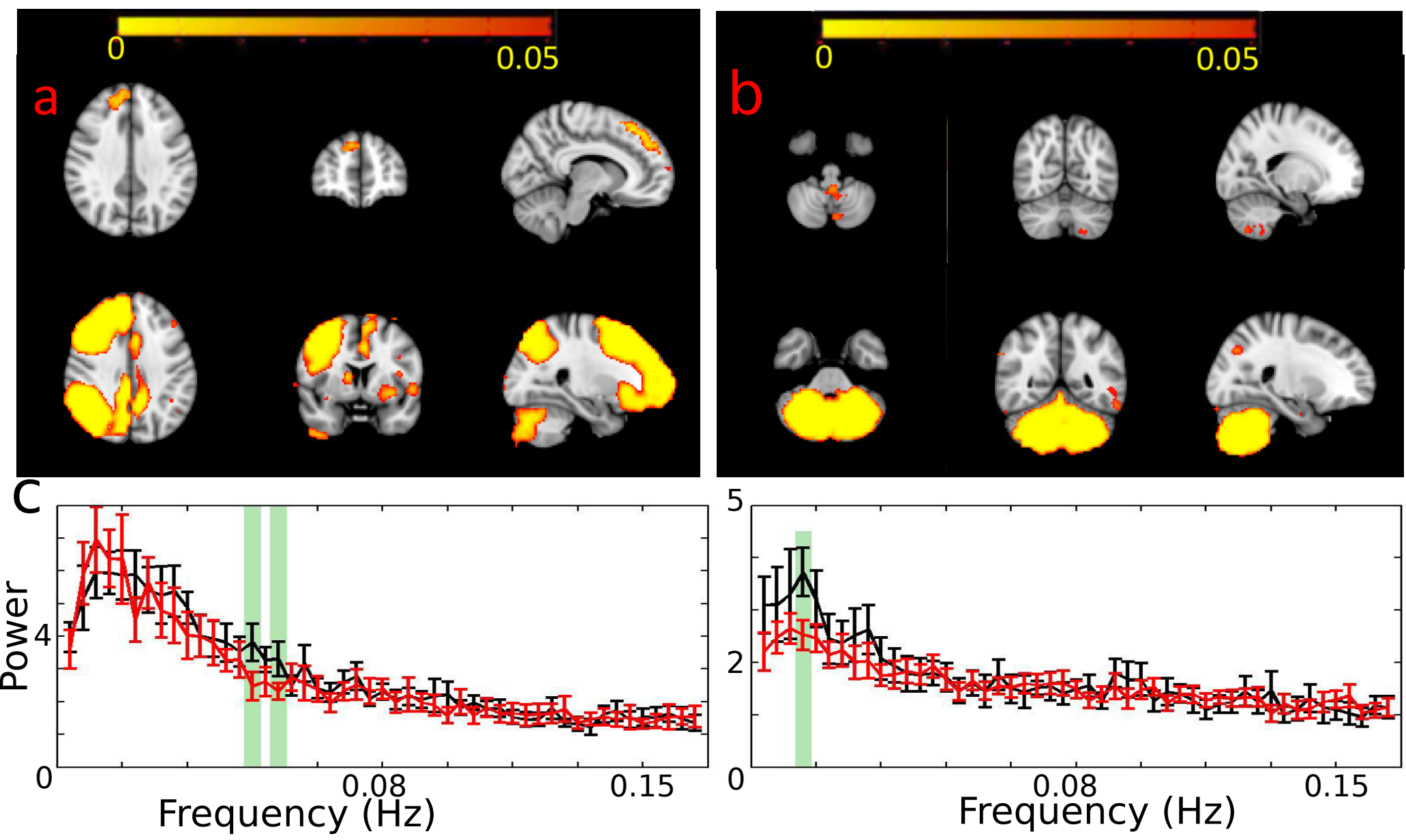


\begin{tabular}{|l|c|c|c|}
\hline & controls & patients & $\mathrm{p}$ \\
\hline $\mathrm{n}$ & 26 & 17 & - \\
\hline gender (male) & 23 & 15 & n.s. \\
\hline age (years) & $37.92 \pm 11.55$ & $37,82 \pm 11.57$ & n.s. \\
\hline handedness (right) & 26 & 17 & n.s. \\
\hline headache side (right) & $\mathrm{NA}$ & 7 & - \\
\hline Disease duration (years) & $\mathrm{NA}$ & $7.7 \pm 6$ & - \\
\hline Average time between bouts (months) & $\mathrm{NA}$ & $12.6 \pm 11.4$ & - \\
\hline Average length of bouts (weeks) & $\mathrm{NA}$ & $4.4 \pm 4.1$ & - \\
\hline cumulative headache days & $\mathrm{NA}$ & $319.19 \pm 243.3$ & - \\
\hline
\end{tabular}




\begin{tabular}{|l|l|l|l|}
\hline Left flipped dataset & $\mathrm{x}(\mathrm{mm})$ & $\mathrm{y}(\mathrm{mm})$ & $\mathrm{z}(\mathrm{mm})$ \\
\hline Left attention network & 50 & 76 & 64 \\
\hline Cerebellar network & 56 & 33 & 9 \\
\hline Right flipped dataset & 40 & 88 & 53 \\
\hline $\begin{array}{l}\text { Right attention network } \\
\text { Cerebellar network }\end{array}$ & 54 & 31 & 11 \\
\hline
\end{tabular}




\section{Click here to access/download
Supplementary Material \\ Supplementary Material Suppl_1.docx}


Click here to access/download Supplementary Material suppl_2_VBM.docx 